\title{
Uncertainty evaluation for the gauge blocks calibration using the modified DMDM gauge block interferometer
}

\author{
V. Zivkovic ${ }^{\star}$, S. Zelenika ${ }^{\star \star}$, and G. Stefanovic ${ }^{\star \star \star}$ \\ Directorate of Measures and Precious Metals, Belgrade, Serbia
}

Received: 20 March 2012 / Accepted: 24 March 2012

\begin{abstract}
This paper presents the evaluation of the uncertainty for the length measurement of gauge blocks by optical interferometry. In order to improve the gauge block calibration method and to decrease measurement uncertainty the old Zeiss-Kösters gauge block interferometer has been modified and improved by DMDM (Directorate of Measures and Precious Metals of Serbia). The main modification of the interferometer covers introduction of two laser sources, as new wavelength standards, as well as new hardware system and interferometer software, developed to completely automate the calibration process. The validation of the new measurement procedure details the uncertainty budget. The measurement method and model equation, which serves as the basis for the uncertainty evaluation is described. The uncertainty evaluation is performed in accordance with the document JCGM 100:2008-Evaluation of measurement data - Guide to the expression of uncertainty in measurement (GUM).
\end{abstract}

Keywords: Uncertainty evaluation; gauge block; interferometry; calibration

\section{Introduction}

Multiple wavelength interferometry is used routinely for length measurements of gauge blocks to the highest accuracy. This technique involves the generation of fringes between a reference surface and the measuring surface of the gauge block under calibration. Measuring of the shift between these fringe fields at a number of known wavelengths allows the unambiguous determination of the length of the gauge block. This technique is known as method of exact fractions and has been routinely carried out at DMDM for many years using Zeiss-Kösters interferometer [1]. In relatively many National Metrology Institutes (NMI) worldwide, this instrument is in routine use for gauge block calibration, so various modifications on the original setup with different approaches were already performed on them.

In order to improve the gauge block calibration method and to decrease measurement uncertainty the old ZeissKösters gauge block interferometer has been modified by DMDM. Validation of the new measurement procedure included also detailed uncertainty budget development. The uncertainty evaluation is performed in accordance with the document JCGM 100:2008-Evaluation of measurement data - Guide to the expression of uncertainty in measurement (GUM) [2].

\footnotetext{
* Correspondence: vidazivkovic@dmdm.rs

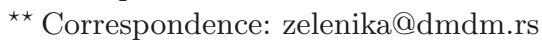

${ }^{\star \star \star}$ Correspondence: gocastef@dmdm.rs
}

\section{Modification of the interferometer}

In order to perform interferometric calibration of gauge blocks with traceability to the definition of the meter using red He-Ne stabilized laser, several modifications were made to the original Zeiss interferometer design in order to decrease the measuring uncertainty and to speed up the measurement process.

The main modification of the interferometer covers introduction of two laser sources, as new wavelength standards, as well as new hardware system and interferometer software, developed to completely automate the calibration process.

Several features of the original Zeiss interferometer were not modified, especially the Kösters prism (used as a monochromator for helium and krypton light sources) and the optical elements inside the interferometer. The quality of original optical elements is unknown, and could be compromised because of contamination and the age of the instrument. Due to the closed architecture of the device, only the summary influence of these elements to the measurement uncertainty could be established.

\section{Hardware modifications}

The overview of the modified DMDM interferometer is presented in Figure 1.

The original light sources in the interferometer were helium and krypton discharge lamps. Values of the wavelengths of these lines were recommended by the CIPM 


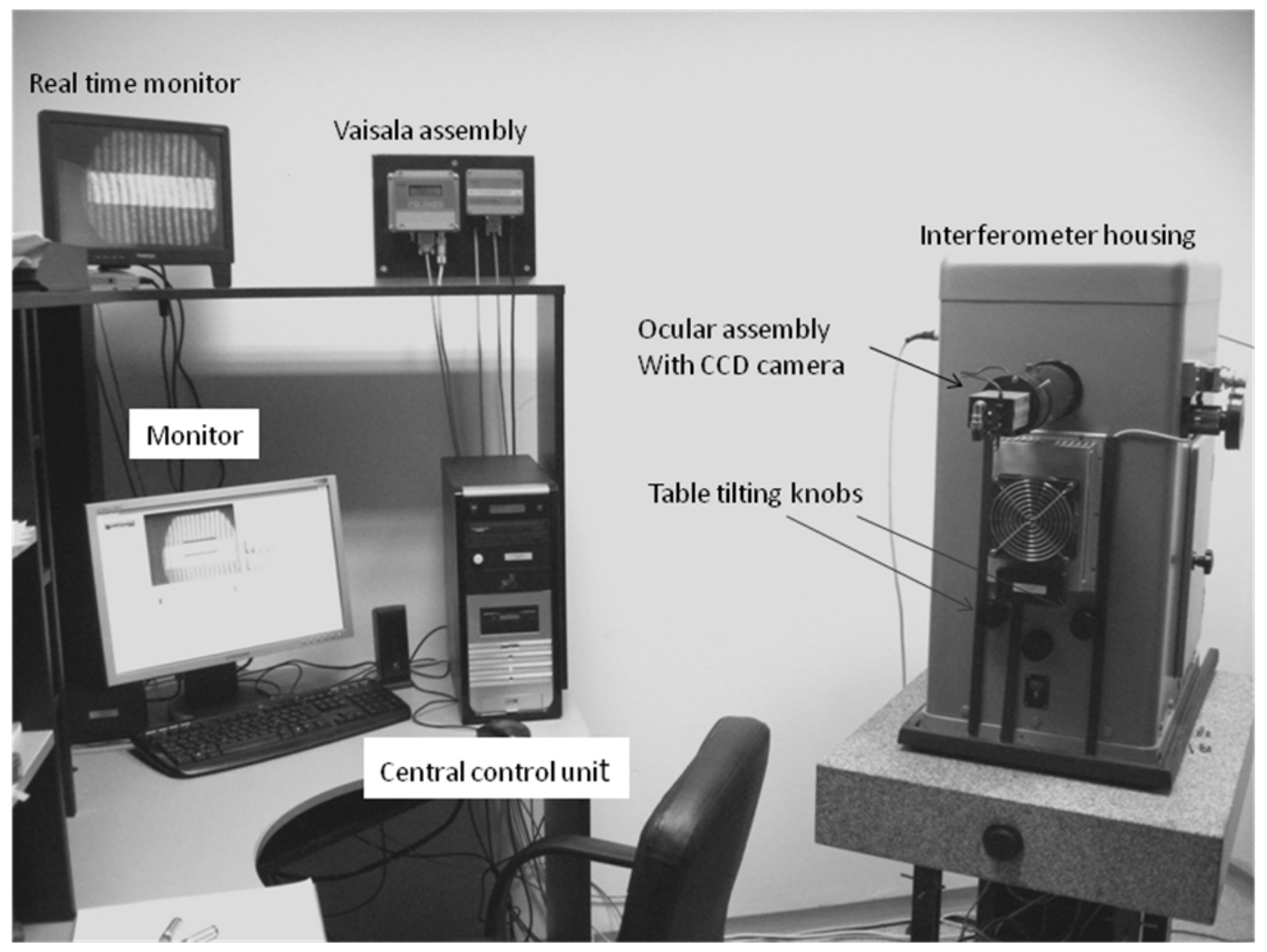

Fig. 1. The overview of the DMDM interferometer.

in 1963 and referenced in the latest mise en pratique and making them traceable to the current definition of the meter. These values are for specific operation conditions, and we do not have the capability to check their actual operating wavelengths. Most of the lines of those sources are now too weak to be easy observed by CCD camera.

Instead of those discharge lamps we introduced stabilized lasers into the interferometer system. Discharge lamps have been replaced with red $(633 \mathrm{~nm})$ and green (543 nm) stabilized helium-neon lasers. In addition to the reference red He-Ne laser, a green He-Ne laser was used as the source of secondary wavelength. We use commercial NPL-Brown\&Sharpe two frequency (Zeeman) stabilized He-Ne lasers as light sources.

The laser sources are coupled to the interferometer through optical fiber. Light from lasers are fed into a multimode optical fiber of $200 \mu \mathrm{m}$ in diameter via a fiber combiner and coupling lens. The other end of the fiber is fixed on the input hole of the interferometer housing and properly positioned with respect to the collimation optics of the interferometer. The fiber end acts as a point source of laser light. In that way, a fiber optic feed supplies a traceable radiation of the stabilized lasers to the interferometer.

The coherence and the intensity of the laser light incident on the gauge block and platen results in a strong speckle pattern obscuring the interference fringes.

Vibrating the fibre restores the fringe pattern. The optical fibre is attached on a small buzzer (loudspeaker) which vibrates enough to eliminate any speckle pattern from the image of the interference fringes by causing the small changes in the refractive index of the fiber and disturbing the spatial coherence of the laser light on a time scale related to the vibration frequency. If this time scale is shorter than the averaging time of the camera, the spatial pattern is not seen.

The interferometer is thermally active: internal temperature is controlled by peltier elements together with slow-turning fan blade. Fan blade gently stirs the air inside the interferometer enclosure to prevent any thermal gradients inside the interferometer. The motor of the fan blade is placed outside the interferometer. Temperature inside the interferometer is kept within $0.1{ }^{\circ} \mathrm{C}$ around $20{ }^{\circ} \mathrm{C}$ during calibration. Anyway, system for active temperature control is capable to keep the temperature within $0.02{ }^{\circ} \mathrm{C}$ around $20{ }^{\circ} \mathrm{C}$ during operation. Normally, during calibration of the each particular gauge block the peltier elements and the fan blade are switched-off.

The internal interferometer temperature of the air and the gauge blocks as well as the air pressure and the relative humidity are measured and monitored with several sensors.

The internal temperature (of the air and the gauge block) is measured using bead-in-glass NTC thermistors (manufacturer YSI) calibrated by Group for temperature at DMDM, instead of old mercury-in-glass thermometers.

Temperature of longer gauge blocks is measured by taking the average of measurements made by two thermistors which are fixed in the small magnetic circular blocks 


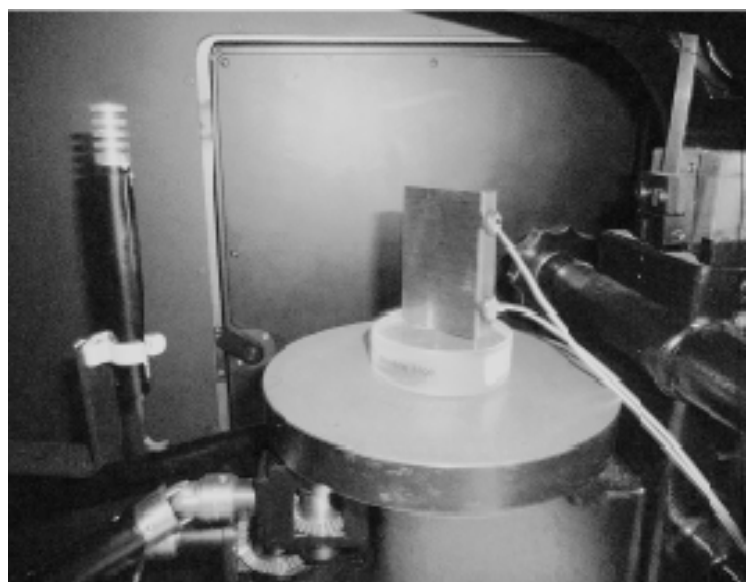

Fig. 2. Temperature measurement of the gauge block during calibration.

and placed to be in direct contact with gauge block under the calibration, as shown in Figure 2.

Temperature of shorter gauge blocks are measured by putting thermistors on another gauge block of similar length and placed near the gauge block under calibration.

The relative humidity is measured using VAISALA HMP45A-P relative humidity and temperature probe calibrated by Group for temperature at DMDM.

Part for temperature measurement of this probe is used only for monitoring thermal gradients in air inside the interferometer during calibration process, not for calculation of the results.

Air pressure is measured using a VAISALA PTU 200 Class A PTU transmitter. This is an improvement over the previous pressure gauge as the new gauge can be calibrated to an expanded uncertainty $(k=2)$ of less than $10 \mathrm{~Pa}$ (in absolute accuracy) compared to the previous mercury barometer and 100 Pa expanded uncertainty.

During calibration the gauge block are wrung onto the quartz optical flat and measured in a vertical orientation. We use Custom Scientific double side optical flats. Flatness of both surfaces of the optical flats is better than $\lambda / 20$ which satisfy the requirements of $0.03 \mu \mathrm{m}$ of the ISO 3650 standard for gauge blocks [3]. Anyway, calibration results (peak-to-valley) of the flats show values for flatness from $\lambda / 22.2$ to $\lambda / 38.5$ for $633 \mathrm{~nm}$ wavelength. Quartz is used so that quality of the wringing can be inspected from the other side of the flat. Diameter of the flats is larger than $5 \mathrm{~cm}$ which is enough to maintain the integrity of the surface flatness of the flat with wrung gauge block on it.

Interference fringe pattern of two lasers is changed by rotating the Kösters prism which act as a monochromator. The rotational prism movement has two positions, one each for the red and the green helium-neon laser. The prism movement is driven by small step motor. This motor receives commands from the computer and rotates the Kösters prism in order to select laser wavelength one after another and to perform automatic measurements. To obtain good and sharp interferometric image the prism can be rotated manually as well.

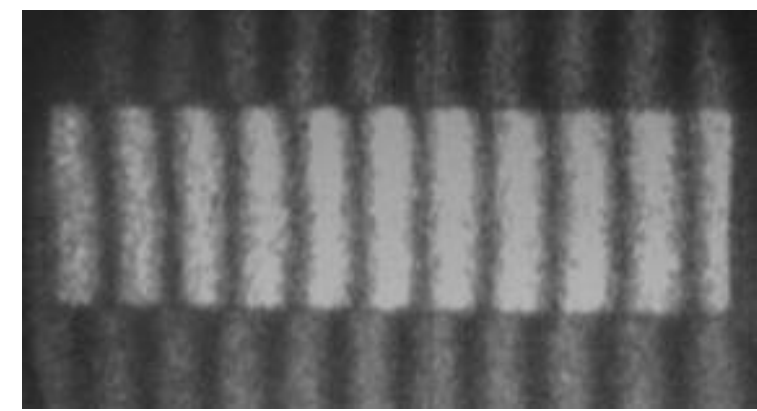

Fig. 3. The fringe pattern.

The interferometer eyepiece formerly used to observe interference fringe pattern has been replaced with monochrome analogue high resolution CCD camera. The specified minimum illumination for this camera is 0.1 lux. CCD camera is placed in the plane of the real image of the fringe pattern.

Figure 3 shows the quality of the interferometric fringe pattern obtained by DMDM interferometer.

\section{Software modification}

The computer is used to control the wavelength selection, to grab and display camera interference image and to analyze the measurement data and calculate gauge block length. Measurement of temperature, pressure and humidity are collected via the $\mathrm{AD} / \mathrm{DA}$ interface card from calibrated instruments and the refractive index of air and the air wavelengths of two lasers are calculated. The camera output is displayed on the computer screen, the crosshairs are manually positioned over the gauge block and the platen and the computer calculates the fringe fraction. This is done for both $633 \mathrm{~nm}$ and $543 \mathrm{~nm}$ wavelength respectively. Real-time interference image is also displayed on the additional monitor that we use during positioning the gauge block in the incident laser light to obtain adequate fringe image.

The computer software is written in Visual $\mathrm{C}++$ program language. The interferometer software screen (user interface) is shown in Figure 4.

The computer software determines the length of each gauge block by applying the method of exact fractions using the measured fractions at two laser wavelengths. Red $\mathrm{He}-\mathrm{Ne}$ laser is used as reference laser. A green He-Ne laser was used as source of secondary wavelength. Traceability of the measuring results goes through red laser radiation.

The vacuum wavelengths of those lasers are adjusted for the refractive index of air in the interferometer using empirical formula given by Edlén and modified by Edlén [4], and Birch and Downs [5,6].

The length of each gauge block is also corrected to account for the material properties of the gauge blocks: thermal properties via thermal expansion coefficient and optical properties by calculating average optical reflection phase-shift. 


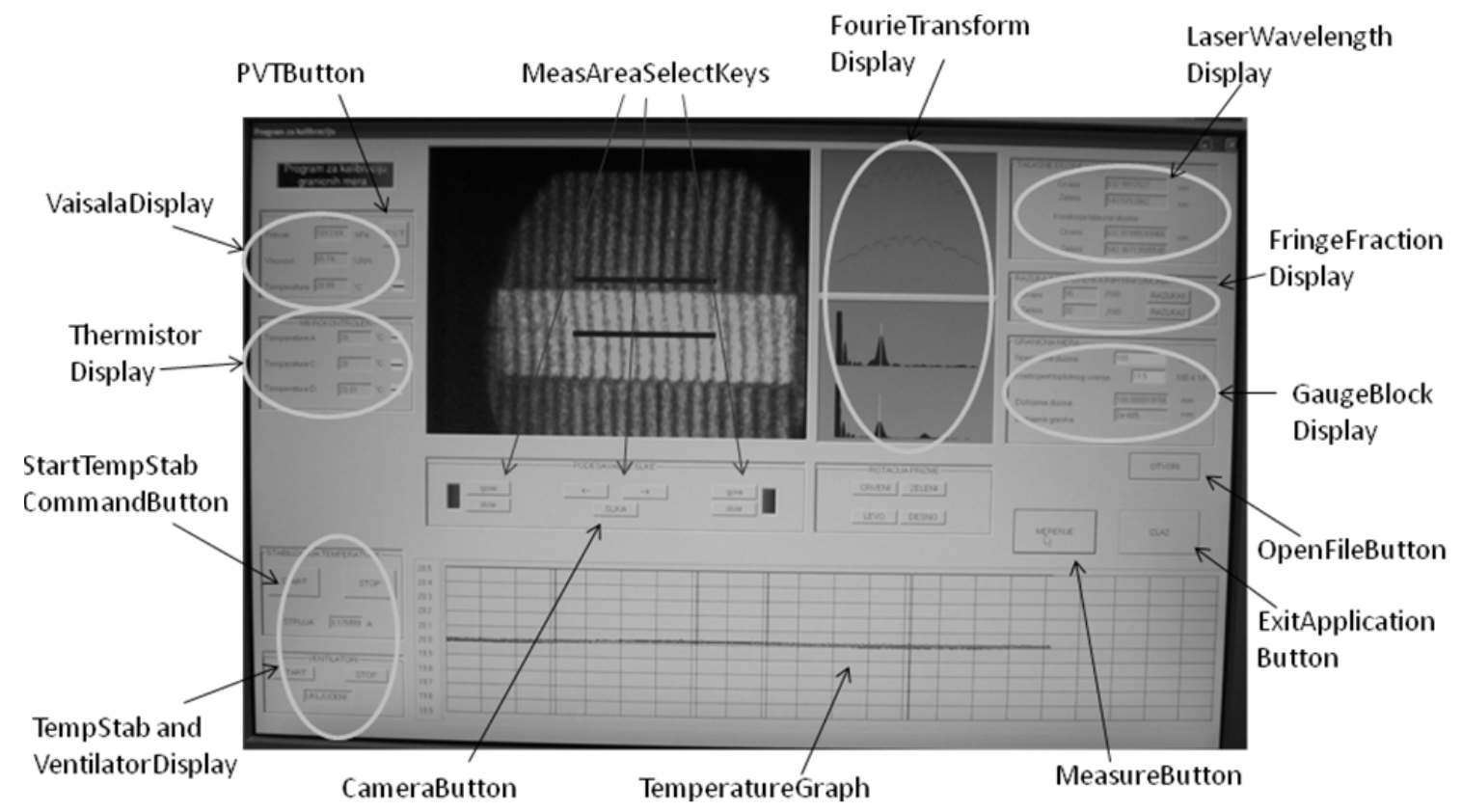

Fig. 4. The interferometer software screen.

Computer calculates fringe fractions using Fourier transform method. All solutions within first three interference fringe orders are calculated. The solution with minimum standard deviation is chosen.

\section{The model equation}

It is convenient to express measured length $l$ of the gauge block of nominal length $L$ measured by interferometry as a deviation from nominal length $d$, because the modern gauge blocks are made within less than few micrometers of their nominal lengths:

$$
d=l-L \text {. }
$$

The model equation include all corrections to the measured value of gauge block length due to influences parameters impacting on measuring results and can be expressed as

$$
d=l_{f i t}-L+l_{t}+l_{w}+l_{A}+l_{\Omega}+l_{n}+l_{G}+l_{\phi} .
$$

where $-l_{f i t}$ is the best-fit solution for gauge block length [7]. $l_{\text {fit }}$ can be expressed as

$$
l_{f i t}=\frac{1}{q} \sum_{i=1}^{q}\left(k_{i}+F_{i}\right) \frac{\lambda_{i}}{2} .
$$

Parameters which has influence on the gauge block length solution are the measured interference fringe fraction $F_{i}$ and the vacuum wavelength $\lambda_{i} . q$ is number of wavelengths used in the measurement ( $633 \mathrm{~nm}$ and $543 \mathrm{~nm})$ and $k_{i}$ is the integer part of the fringe order.

$-L$ is the nominal length of the gauge block. It is assumed that associated standard uncertainty $u(L)$ is zero; $-l_{t}$ is the gauge block temperature correction which arises from gauge block temperature offset from $20^{\circ} \mathrm{C} . l_{t}$ can be expressed as

$$
l_{t}=\theta \alpha L=\left(20-t_{g}\right) \alpha L .
$$

Influence parameters on this correction are the gauge block temperature (in degrees Celsius) $t_{g}$ and thermal dilatation coefficient for the gauge block material $\alpha$.

$-l_{w}$ is the correction attributed to the thickness of the wringing film due to the wringing action;

$-l_{A}$ is the correction attributed to the wave-front errors due to imperfect interferometer optics;

$-l_{\Omega}$ is the obliquity correction and can be expressed as

$$
l_{\Omega}=\Omega L=\left(\frac{a^{2}}{16 f^{2}}+\frac{x^{2}}{2 f^{2}}\right) L .
$$

This correction is dependent on the collimator lens focal length $f$, aperture diameter $a$ and the lateral offset $x$.

$-l_{n}$ is the refractive index correction and can be expressed as

$$
l_{n}=(n-1) L,
$$

$n$ is the refractive index of air evaluated by modified Edlén formula;

- $l_{G}$ is the geometry correction accounting for nonflatness and non-parallelism of the gauge block;

$-l_{\phi}$ is the correction attributed to the phase change due to difference in material and/or surface texture between the gauge block and the platen and can be expressed as

$$
l_{\phi}=\frac{1}{m-1}\left(l_{p}-\sum_{i=1}^{m} l_{i}\right)
$$

$l_{p}$ represent the measured length of the pack and $l_{i}$ the measured length of the $m$ individual gauge blocks making up the pack 


\section{Equation of the combined standard uncertainty}

Following the propagation law of uncertainty, the combined standard uncertainty $u_{c}$ of the measured gauge block length $l$ is:

$$
\begin{aligned}
u_{c}^{2}= & u\left(l_{f i t}\right)^{2}+u\left(l_{t}\right)^{2}+u\left(l_{w}\right)^{2}+u\left(l_{A}\right)^{2} \\
& +u\left(l_{\Omega}\right)^{2}+u\left(l_{n}\right)^{2}+u\left(l_{G}\right)^{2}+u\left(l_{\phi}\right)^{2}
\end{aligned}
$$

\section{Uncertainty evaluation}

The measurement uncertainty is evaluated following the rules of the Guide to the Expression of the Uncertainty in Measurement or GUM. In the terminology of the GUM, statistical evaluation of the uncertainty groups the uncertainty components as type $\mathrm{A}$ and all others as type B. The combined standard uncertainty is the sum of squared standard uncertainty components. Each component represents the standard uncertainty associated to each factor that has influence on the measurement results. The influence parameters and the associated standard uncertainties listed in Table 1. are grouped into two groups. Length dependent and end effect uncertainties are summed separately in quadrature and combined into the expanded uncertainty $U=k u_{c}$, where $k=2$, in the end of the evaluation process.

\subsection{Fringe fraction measurement}

The fringe fractions are calculated using Fourier transform method. The red (green) fringe fractions are determined on the basis of five measurements for each wavelength as the average value. The nominal order of interference is calculated for the red wavelength. The observed fringe fractions are used to calculate the length of the gauge block and green residue. All solutions within first three interference fringe orders are calculated. The solution with minimum standard deviation is chosen.

The standard uncertainty in fringe fraction reading has been determined experimentally to be 0.01 fringe taken from 30 repeated readings

\subsection{Thermal effects}

The standard uncertainty associated to temperature influence on gauge block length includes standard uncertainties that arise from gauge block temperature measurements and known of the value of the thermal expansion coefficient.

In uncertainty evaluation due to measurement of the gauge block temperature the combined standard uncertainty is used. The combined standard uncertainty is consist of two components. One is attributed to the temperature gradient of the gauge block during calibration. In the uncertainty evaluation we use that gradient is $10 \mathrm{mK}$ as rectangular distribution. Actual situation during calibration shows gradients of about $(2-3) \mathrm{mK}$. We use some overestimated value for safety. Second component is combined standard uncertainty that is attributed to gauge block temperature measurements by thermistors and includes components for traceable calibration, digital resolution of the thermistors reading and drift between calibrations. This component is estimated to be about $10 \mathrm{mK}$. Standard uncertainty of calibration is $5 \mathrm{mK}$ (normal distribution), standard uncertainty of resolution is about $3 \mathrm{mK}$ (due to $10 \mathrm{mK}$ reading capability, rectangular distribution). Eight years calibration history of two thermistors shows the total drift of about $70 \mathrm{mK}$ in worst case (for second thermistor the eight-year drift is about $40 \mathrm{mK}$ ) during entire period, which leads to less than $10 \mathrm{mK}$ per year drift. This is a rectangular distribution that gives (after dividing by square root of 3 ) about $6 \mathrm{mK}$ for one year drift. Total combined uncertainty of $10 \mathrm{mK}$ is used for uncertainty calculation.

In our uncertainty budget evaluation we used that gauge block is made of steel with thermal dilatation coefficient of $11.5 \times 10^{-6} \mathrm{~K}^{-1}$ and associated uncertainty of $1 \times 10^{-6} \mathrm{~K}^{-1}$.

\subsection{Wringing film}

The expectation value of this correction is zero because the interferometric (optical) length of the gauge block includes and that wringing film by the definition. Because of the variation of the thickness of the wringing film the associated standard uncertainty is non-zero. The associated standard uncertainty is determined experimentally by repeated measurements (including re-wringing) for steel gauge blocks of nominal length of $3 \mathrm{~mm}$ and $5 \mathrm{~mm}$ and found to be about $7 \mathrm{~nm}$.

\subsection{Wavefront errors}

Correction attributed to the wave-front errors arise from imperfect interferometer optics. Expectation value of this correction is zero, but the standard uncertainty is nonzero. This standard uncertainty is determined experimentally with platen without a gauge block but with a wire placed onto platen to represent the outline of the gauge blocks. Repeated measurements show that the standard uncertainty is about $3 \mathrm{~nm}$ (rectangular distribution).

\subsection{Obliquity}

The obliquity correction accounting shifts in phase of laser light due to optical design and alignment properties of the interferometer. The value used in uncertainty calculations is $200 \mu \mathrm{m}$ for the fibre (aperture) diameter with the associated standard uncertainty of $5 \mu \mathrm{m}$ and the lens focal length of about $250 \mathrm{~mm}$ with the associated standard uncertainty of $0.15 \mathrm{~mm}$. 
Table 1. The uncertainty budget.

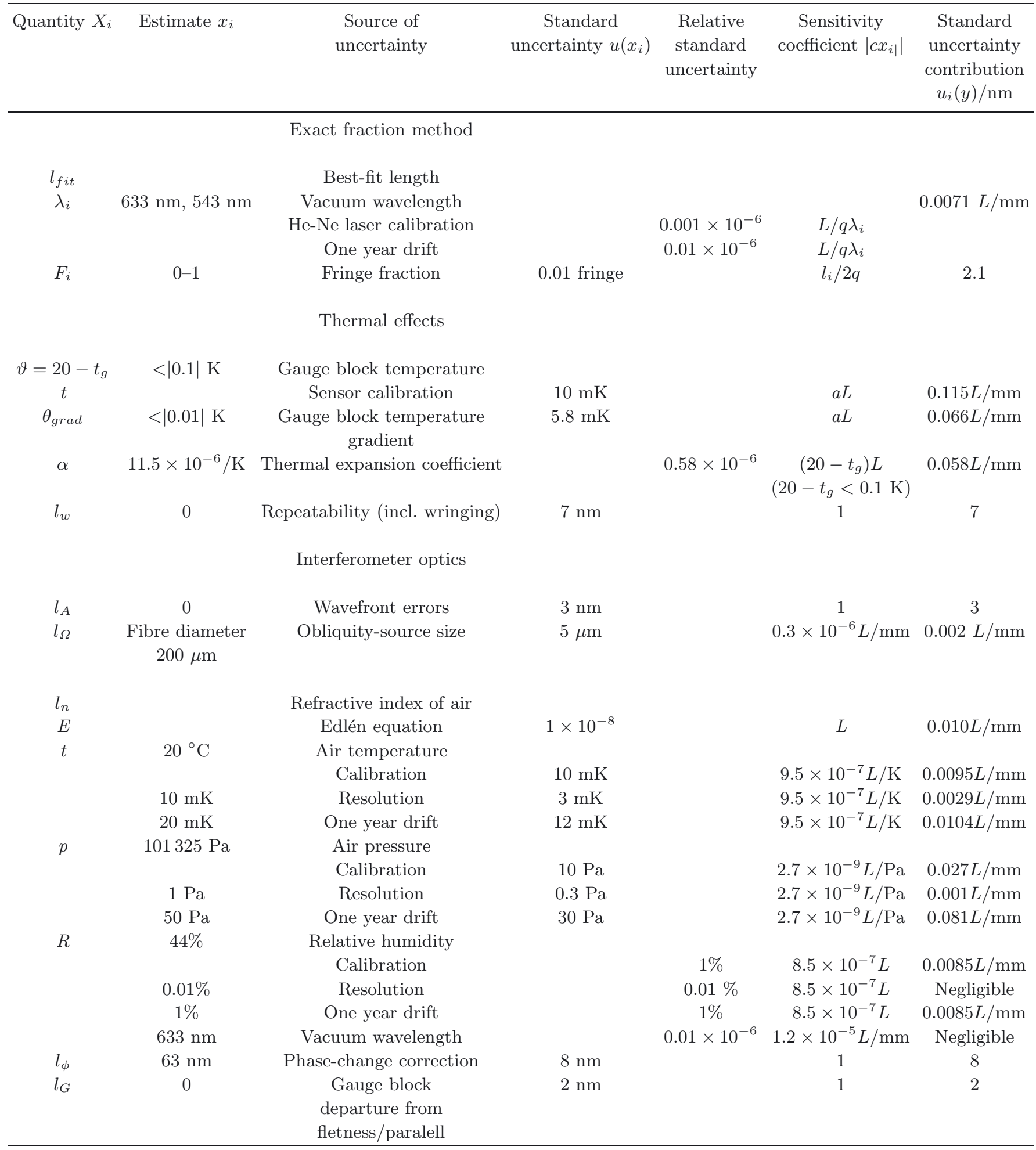

Combined standard uncertainty: $u^{2}(L)=\left(11.422+0.1702(L / \mathrm{mm})^{2}\right) \mathrm{nm}^{2}$

Linearized expanded uncertainty for coverage factor $k=2: U(L)=(23+0.18(L / \mathrm{mm})) \mathrm{nm}$

Calculation of standard uncertainty components for gauge blocks measurement by optical interferometry steel gauge blocks $a=(11.5+/-1) 10 \mathrm{E}-06 / \mathrm{K}$. 


\subsection{Refractive index of air}

The refractive index of air is evaluated by modified Edlén formula. Associated standard uncertainty includes empirical fit of the Edlén model, the environment air density factors of temperature, pressure and humidity as well as the vacuum wavelength of the laser light.

Standard uncertainties attributed to the measurement of air temperature, pressure and relative humidity are combined standard uncertainties and includes components for traceable calibration, digital reading capability and drift between calibrations.

For air temperature measurement the standard uncertainty of calibration is $10 \mathrm{mK}$ (normal distribution), standard uncertainty of resolution is about $3 \mathrm{mK}$ (due to $10 \mathrm{mK}$ reading capability, rectangular distribution). Four years calibration history of thermistor shows the total drift of about $75 \mathrm{mK}$ during entire period, which leads to less than $20 \mathrm{mK}$ per year drift. This is rectangular distribution that gives (after dividing by square root of 3 ) about $12 \mathrm{mK}$ for one year drift.

For air pressure measurements the standard uncertainty of calibration is $10 \mathrm{~Pa}$ (normal distribution), standard uncertainty of resolution is about $0.3 \mathrm{~Pa}$ (due to $1 \mathrm{~Pa}$ reading capability, rectangular distribution). When the uncertainty budget is made we had not repeated calibration of the pressure transmitter due to objective reasons and we used value for one-year drift to be $50 \mathrm{~Pa}$ with rectangular distribution. That value is overestimated and we used it for safety reasons. In the meantime two pressure transmitter calibrations were performed and the results show total drift over the period of six years of only $1 \mathrm{~Pa}$.

For air humidity measurements the standard uncertainty of calibration is $1 \%$ (normal distribution), standard uncertainty of resolution is about $0.0003 \%$ (due to $0.01 \%$ reading capability, rectangular distribution) and is negligible. Six years calibration history of humidity sensor shows the total drift of about 3\% during entire period, which leads to less than $1 \%$ per year drift. This is rectangular distribution and we use slightly overestimated value for uncertainty calculation.

\subsection{Phase-change correction}

The phase-change correction is accounting for the difference between optical length of gauge block and its mechanical length. The combined standard uncertainty attributed to the phase change correction is determined experimentally using stack (pack) experiment with four steel gauge blocks and the evaluated uncertainty from Table 1. The gauge block used in this measurements are small (with nominal values less than $5 \mathrm{~mm}$ ) to eliminate temperature effect on the result of measurements. The associated standard uncertainty is found to be about $8 \mathrm{~nm}$.

\section{Calculation of the expanded uncertainty}

The expanded uncertainty, $U$, is obtained by multiplying the combined standard uncertainty to the coverage factor $k$,

$$
U=k u_{c}
$$

where $k=2$, for $95 \%$ confidence level.

Based on the above analysis the total expanded uncertainty of the modified Zeiss-Kösters interferometer is found to be:

$$
U^{2}(L)=\left(23^{2}+0.34^{2}(L / \mathrm{mm})^{2}\right) \mathrm{nm}^{2}
$$

or in linearized form for measurements taken at $1 \mathrm{~mm}$ and another measurement taken at $100 \mathrm{~mm}$ :

$$
U(L)=(23+0.18(L / \mathrm{mm})) \mathrm{nm},
$$

where $L$ is nominal length of the gauge block.

The expanded uncertainty (for $k=2$ ) of the nonmodified Zeiss-Kösters interferometer was

$$
U(L)=(30+0.30(L / \mathrm{mm})) \mathrm{nm},
$$

in linearized approximation, where $L$ is the nominal length of the gauge block.

From above results for expanded uncertainty it is obvious that the value for end-effect uncertainty decreased for $7 \mathrm{~nm}$ and the value for length depending uncertainty decreased for $12 \mathrm{~nm}$.

Main contribution to the lower total uncertainty has been made in the uncertainty components attributed to the fringe fraction measurement and analysis, evaluation of the refractive index of air and ambient wavelength, and gauge block temperature measurement.

\section{Validation of the modified interferometer system}

Internal validation at DMDM was performed using our Alan Browne gauge blocks of grade $\mathrm{K}$ which have calibration certificate issued by Belgium NMI, SMD. Those preliminary results were quite satisfactory.

At the international level, validation of the modified gauge block interferometer was performed through bilateral comparison. The bilateral interlaboratory comparison concerning the central length measurements of gauge blocks by interferometry between the dimensional laboratory of the Directorate of Measures and Precious Metals (DMDM), Serbia and the dimensional laboratory of the Hellenic Institute of Metrology, Greece was performed during 2010.

The technical protocol of the comparison was made according to the key comparison EUROMET.L-K1.1, calibration of gauge blocks by interferometry, 2004 [8].

The length of the gauge blocks involved in the comparison were $5 \mathrm{~mm}, 20 \mathrm{~mm}, 50 \mathrm{~mm}, 75 \mathrm{~mm}, 100 \mathrm{~mm}$, and they was made of steel.

Results of the bilateral comparison are shown in Table $2[9]$. 
Table 2. Results of the comparison.

\begin{tabular}{|c|c|c|c|}
\hline $5 \mathrm{~mm}$ & EIM 1 & MDM & EIM 2 \\
\hline$\Delta l(\mu \mathrm{m})$ & 0.002 & 0.012 & 0.008 \\
\hline$U c(\Delta l)(k=2)(\mu \mathrm{m})$ & 0.025 & 0.023 & 0.025 \\
\hline$\Delta l_{\text {mean }}(\mu \mathrm{m})$ & 0.008 & & \\
\hline$U\left(\Delta l_{\text {mean }}\right)(\mu \mathrm{m})$ & 0.014 & & \\
\hline$d=\Delta l-\Delta l_{\text {mean }}(\mu \mathrm{m})$ & -0.006 & 0.004 & 0.000 \\
\hline$U(d)(\mu \mathrm{m})$ & 0.021 & 0.018 & 0.021 \\
\hline$E j=d / U(d)$ & -0.27 & 0.24 & 0.02 \\
\hline $20 \mathrm{~mm}$ & EIM 1 & $\overline{D M D M}$ & EIM 2 \\
\hline$\Delta l(\mu \mathrm{m})$ & 0.023 & 0.019 & 0.012 \\
\hline$U c(\Delta l)(k=2)(\mu \mathrm{m})$ & 0.026 & 0.024 & 0.026 \\
\hline$\Delta l_{\text {mean }}(\mu \mathrm{m})$ & 0.018 & & \\
\hline$U\left(\Delta l_{\text {mean }}\right)(\mu \mathrm{m})$ & 0.015 & & \\
\hline$d=\Delta l-\Delta l_{\text {mean }}(\mu \mathrm{m})$ & 0.005 & 0.001 & -0.006 \\
\hline$U(d)(\mu \mathrm{m})$ & 0.022 & 0.019 & 0.022 \\
\hline$E j=d / U(d)$ & 0.23 & 0.05 & -0.28 \\
\hline $50 \mathrm{~mm}$ & EIM 1 & DMDM & EIM 2 \\
\hline$\Delta l(\mu \mathrm{m})$ & 0.065 & 0.063 & 0.065 \\
\hline$U c(\Delta l)(k=2)(\mu \mathrm{m})$ & 0.032 & 0.028 & 0.032 \\
\hline$\Delta l_{\text {mean }}(\mu \mathrm{m})$ & 0.064 & & \\
\hline$U\left(\Delta l_{\text {mean }}\right)(\mu \mathrm{m})$ & 0.018 & & \\
\hline$d=\Delta l-\Delta l_{\text {mean }}(\mu \mathrm{m})$ & 0.001 & -0.001 & 0.001 \\
\hline$U(d)(\mu \mathrm{m})$ & 0.027 & 0.022 & 0.027 \\
\hline$E j=d / U(d)$ & 0.03 & -0.06 & 0.03 \\
\hline $75 \mathrm{~mm}$ & EIM 1 & DMDM & EIM 2 \\
\hline$\Delta l(\mu \mathrm{m})$ & 0.144 & 0.124 & 0.142 \\
\hline$U c(\Delta l)(k=2)(\mu \mathrm{m})$ & 0.04 & 0.034 & 0.04 \\
\hline$\Delta l_{\text {mean }}(\mu \mathrm{m})$ & 0.135 & & \\
\hline$U\left(\Delta l_{\text {mean }}\right)(\mu \mathrm{m})$ & 0.022 & & \\
\hline$d=\Delta l-\Delta l_{\text {mean }}(\mu \mathrm{m})$ & 0.009 & -0.011 & 0.007 \\
\hline$U(d)($ mum $)$ & 0.034 & 0.026 & 0.034 \\
\hline$E j=d / U(d)$ & 0.26 & -0.43 & 0.20 \\
\hline $100 \mathrm{~mm}$ & EIM 1 & DMDM & EIM 2 \\
\hline$\Delta l(\mu \mathrm{m})$ & 0.138 & 0.103 & 0.136 \\
\hline$U c(\Delta l)(k=2)(\mu \mathrm{m})$ & 0.048 & 0.041 & 0.048 \\
\hline$\Delta l_{\text {mean }}(\mu \mathrm{m})$ & 0.123 & & \\
\hline$U\left(\Delta l_{\text {mean }}\right)(\mu \mathrm{m})$ & 0.026 & & \\
\hline$d=\Delta l-\Delta l_{\text {mean }}(\mu \mathrm{m})$ & 0.015 & -0.020 & 0.013 \\
\hline$U(d)(\mu \mathrm{m})$ & 0.040 & 0.032 & 0.040 \\
\hline$E j=d / U(d)$ & 0.37 & -0.64 & 0.32 \\
\hline
\end{tabular}

where:

- $\Delta l$ (in $\mu \mathrm{m})$ is measured deviation from the nominal length of the gauge block;

- $U c(\Delta l)(k=2)$ (in $\mu \mathrm{m})$ is the expanded uncertainty of the measured deviation for $\sim 95 \%$ confidence level;

- $\Delta l_{\text {mean }}$ (in $\mu \mathrm{m}$ ) is the reference value $\left(x_{r e f}\right)$ of the deviation of the central length from its nominal value and it was determined using the results of all the measurements reported from both laboratories and evaluating their weighted mean;

- $U\left(\Delta l_{\text {mean }}\right)(k=2)$ (in $\left.\mu \mathrm{m}\right)$ is the expanded uncertainty of the reference value for $\sim 95 \%$ confidence level;
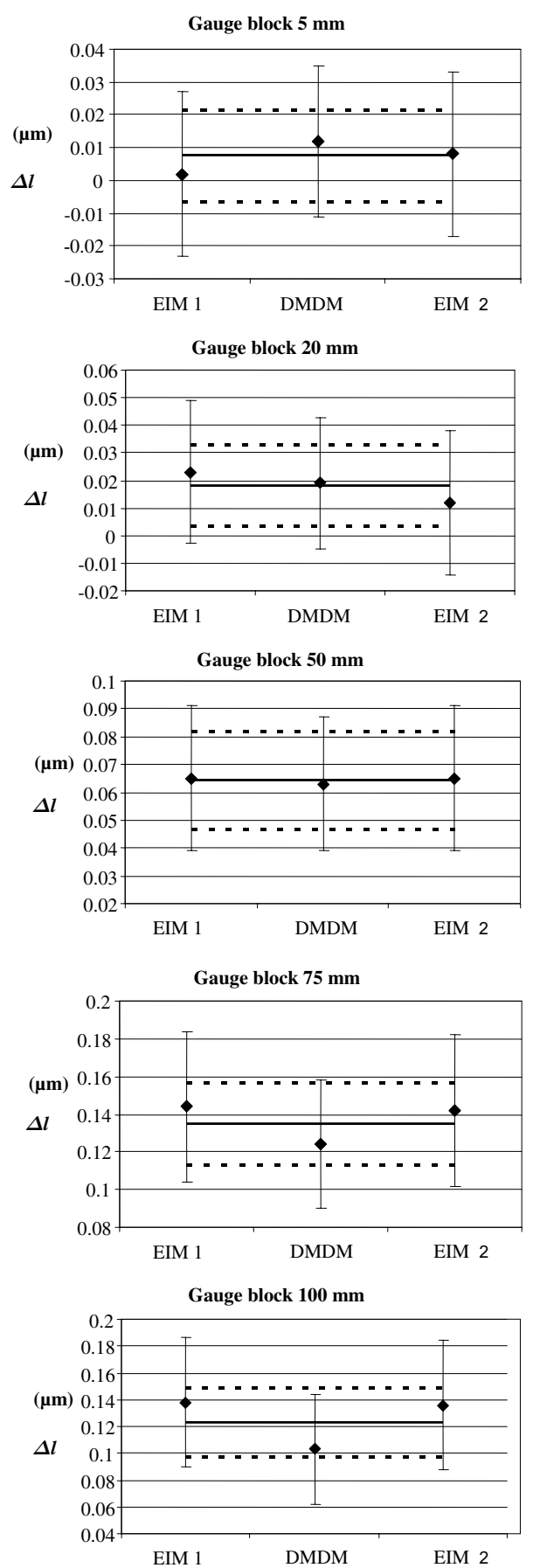

Fig. 5. Graphical presentation of the comparison results.

$-d=x_{i}-x_{r e f}=\Delta l-\Delta l_{\text {mean }}$ (in $\mu \mathrm{m}$ ) is the difference between measured deviation of both participants and reference deviation;

- $U(d)(k=2)$ (in $\mu \mathrm{m})$ is the expanded uncertainty for the difference $d$ for $\sim 95 \%$ confidence level;

- $E j=d / U(d)$ is $E n$ (Chi-squared) value that represent the degree of equivalence between the measurement results of the comparison

Comparison results are shown in the graphical form in Figure 5 as well. 
In Figure 5 with solid and dashed lines are shown the reference value $\left(\Delta l_{\text {mean }}\right)$ and the expanded uncertainty of the reference value $\pm U\left(\Delta l_{\text {mean }}\right)(k=2)$ respectively. The expanded uncertainty of each measurement result $\left( \pm U_{i}\right)$ is shown with the error bars.

The results of the comparison show that all values of the En are less than 1; therefore the consistency test is satisfied and no corrective actions need to be taken. In the other words, DMDM successfully made modifications on its gauge block interferometer.

\section{Conclusion and future trends}

The presented modifications of the DMDM gauge block interferometer shows that modifications are made successfully. The changes allow automatic acquisition and analysis of the interference images, quick determination of the fringe fractional order, automatic reading of the environment parameters and gauge block temperature, and automatic calculation of the gauge block deviation with higher accuracy. The measurement uncertainty of the modified interferometer was evaluated with significant improvements over the original system.

Further works on the interferometer will be focused on introducing the phase-stepping method in the procedure for determination the fringe fractional orders and further decreasing of the measuring uncertainty with decreasing the calibration uncertainty and better drift estimation of the environmental sensors. Future works will be focused on changing the computer software in order to automatize measurements of the flatness and parallelism of the gauge block measuring faces as well.

\section{References}

1. C.F. Bruce, A Kösters-type interferometer, J. Sci. Instr. 33, 478-482 (1956)

2. GUM: Guide to the Expression of Uncertainty in Measurement, JCGM100:2008

3. ISO3650:1998(E), Geometrical Product Specifications (GPS) - Length standards - Gauge blocks

4. B. Edlén, The refractive index of air, Metrologia 2, 71-80 (1966)

5. K.P. Birch, M.J. Downs, An updated Edlén's equation for the refractive index of air, Metrologia 30, 155-162 (1993)

6. K.P. Birch, M.J. Downs, Correction to the updated Edlen equation for the refractive index of air, Metrologia 3, 315$316(1994)$

7. J.E. Decker, J.R. Pekelsky, Uncertainty evaluation for the measurement of gauge blocks by optical interferometry, Metrologia 34, 479-493 (1997)

8. C. Bandis, Bilateral Inter-laboratory Comparison, EIM_DIM_4, Measurement of gauge blocks by interferometry, Measurand: Central Length, Technical Protocol, EIM and DMDM, 2010

9. C. Bandis, Bilateral Inter-laboratory Comparison, EIM_DIM_4, Measurement of gauge blocks by interferometry, Measurand: Central Length, Final Report, EIM and DMDM, 2010 